\title{
Fixed point theorems for multivalued maps via new auxiliary function
}

\author{
Muhammad Usman Ali ${ }^{\mathrm{a}}$, Calogero Vetro ${ }^{\mathrm{b}, 1}$

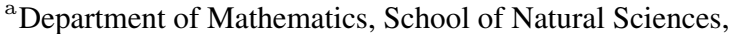 \\ National University of Sciences and Technology, \\ H-12, Islamabad, Pakistan \\ muh_usman_ali@yahoo.com \\ ${ }^{\mathrm{b}}$ Department of Mathematics and Computer Sciences, \\ University of Palermo, \\ Via Archirafi 34, Palermo, 90123, Italy \\ calogero.vetro@unipa.it
}

Received: July 3, 2015 / Revised: January 22, 2016 / Published online: December 2, 2016

Abstract. We introduce a contractive condition involving new auxiliary function and prove a fixed point theorem for closed multivalued maps on complete metric spaces. An example and an application to integral equation are given in support of our findings.

Keywords: $\alpha$-admissible map, fixed point, metric space.

\section{Introduction and preliminaries}

The study of fixed points for multivalued maps is a very important part of fixed point theory. Almost all the major fixed point theorems for self-maps have been extended to multivalued maps. Nadler [21] started this direction in metric fixed point theory. $\mathrm{He}$ used Hausdorff metric to extend Banach contraction principle to multivalued maps. Later on, many authors extended and introduced many other fixed point theorems in different settings, see, for example, [2-7,9-12,15,17-21,23]. Recently, Samet et al. [24] introduced the notions of $\alpha-\psi$-contractive and $\alpha$-admissible self-maps and obtained some interesting fixed point theorems. The extensions of these notions to multivalued maps are available in $[2-7,12,14,18,20]$. Here, we give another generalization of results in [24] to multivalued maps. Then many contractive conditions and related fixed point theorems can be obtained from our result due to the specific family of functions introduced in this paper. An example and an application to integral equation are given to support the theory.

In the following lines, we present some notational and terminological conventions, which will be used throughout this paper. Let $(X, d)$ be a metric space. We denote by $N(X)$ the family of all nonempty subsets of $X$, by $C L(X)$ the family of all nonempty

\footnotetext{
${ }^{1}$ The author was partially supported by INdAM/GNAMPA Project 2015.
} 
closed subsets of $X$ and by $C B(X)$ the family of all nonempty closed and bounded subsets of $X$. For $A \in N(X)$ and $x \in X, D(x, A)=\inf \{d(x, a): a \in A\}$. For every $A, B \in C L(X)$, let

$$
H(A, B)= \begin{cases}\max \left\{\sup _{x \in A} D(x, B), \sup _{y \in B} D(y, A)\right\} & \text { if the maximum exists; } \\ \infty & \text { otherwise. }\end{cases}
$$

Such a function $H$ is called generalized Hausdorff metric induced by $d$. We give few definitions and result due to Asl et al. [7] and Mohammadi et al. [20] for convenience.

Definition 1. (See [7,20].) Let $(X, d)$ be a metric space and $\alpha: X \times X \rightarrow[0, \infty)$ be a function. Then

(i) A map $T: X \rightarrow C L(X)$ is $\alpha_{*}$-admissible if $\alpha(x, y) \geqslant 1 \Rightarrow \alpha_{*}(T x, T y) \geqslant 1$, where $\alpha_{*}(T x, T y)=\inf \{\alpha(a, b): a \in T x, b \in T y\}$.

(ii) A map $T: X \rightarrow C L(X)$ is $\alpha$-admissible if for each $x \in X$ and $y \in T x$ with $\alpha(x, y) \geqslant 1$, we have $\alpha(y, z) \geqslant 1$ for all $z \in T y$.

It is easy to see that $\alpha_{*}$-admissible maps are also $\alpha$-admissible maps, but the converse may not be true as shown in [22, Ex. 15].

Let $\psi:[0, \infty) \rightarrow[0, \infty)$ be a strictly increasing function such that $\sum_{n=1}^{\infty} \psi^{n}(t)<\infty$ for all $t \geqslant 0$. We denote the class of such functions by $\Psi$. Recall that previous condition implies $\psi(t)<t$ for all $t>0$.

Definition 2. (See [7].) Let $(X, d)$ be a metric space. A map $T: X \rightarrow C L(X)$ is called $\alpha_{*}-\psi$-contractive if there exist two functions $\alpha: X \times X \rightarrow[0, \infty)$ and $\psi \in \Psi$ such that

$$
\alpha_{*}(T x, T y) H(T x, T y) \leqslant \psi(d(x, y))
$$

for all $x, y \in X$.

Theorem 1. (See [7].) Let $(X, d)$ be a complete metric space, $\alpha: X \times X \rightarrow[0, \infty)$ be a function, $\psi \in \Psi$ and $T: X \rightarrow C L(X)$ be an $\alpha_{*}$-admissible and $\alpha_{*}$ - $\psi$-contractive map. Suppose that there exist $x_{0} \in X$ and $x_{1} \in T x_{0}$ such that $\alpha\left(x_{0}, x_{1}\right) \geqslant 1$. Assume that if $\left\{x_{n}\right\}$ is a sequence in $X$ such that $\alpha\left(x_{n}, x_{n+1}\right) \geqslant 1$ for all $n \in \mathbb{N}$ and $x_{n} \rightarrow x$ as $n \rightarrow \infty$, then $\alpha\left(x_{n}, x\right) \geqslant 1$ for all $n$. Then $T$ has a fixed point.

\section{Main result}

We start this section by introducing the following class of functions. For other results in this direction, see [8]. We denote by $\Phi$ the family of functions $\phi:[0, \infty)^{4} \rightarrow[0, \infty)$ satisfying the following conditions:

(i) $\phi$ is continuous and nondecreasing in each coordinate;

(ii) Let $u_{1}, u_{2}, w \in[0, \infty)$, if $u_{1}<u_{2}, u_{1}<q w$ for any $q>1$ and $w \leqslant \phi\left(u_{2}, u_{2}\right.$, $\left.u_{1}, u_{2}\right)$, then there exists $\psi \in \Psi$ such that $w \leqslant \psi\left(u_{2}\right)$; 
(iii) Let $u_{1}, u_{2}, w \in[0, \infty)$, if $u_{1} \geqslant u_{2}, u_{1} \leqslant q w$ for any $q>1$ and $w \leqslant \phi\left(u_{1}, u_{2}\right.$, $\left.u_{1}, u_{1}\right)$, then $w=0$;

(iv) Let $u \in[0, \infty)$, if $u \leqslant \phi(0,0, u, u / 2)$ or $u \leqslant \phi(0, u, 0, u / 2)$, then $u=0$.

The following are some examples of functions, which belong to $\Phi$ :

1. $\phi_{1}\left(u_{1}, u_{2}, u_{3}, u_{4}\right)=k \max \left\{u_{1}, u_{2}, u_{3}, u_{4}\right\}$, where $k \in[0,1)$;

2. $\phi_{2}\left(u_{1}, u_{2}, u_{3}, u_{4}\right)=k u_{4}$, where $k \in[0,1)$;

3. $\phi_{3}\left(u_{1}, u_{2}, u_{3}, u_{4}\right)=k \max \left\{u_{1}, u_{2}, u_{3}\right\}$, where $k \in[0,1)$;

4. $\phi_{4}\left(u_{1}, u_{2}, u_{3}, u_{4}\right)=k \max \left\{u_{2}, u_{3}\right\}$, where $k \in[0,1)$;

5. $\phi_{5}\left(u_{1}, u_{2}, u_{3}, u_{4}\right)=k u_{1}$, where $k \in[0,1)$;

6. $\phi_{6}\left(u_{1}, u_{2}, u_{3}, u_{4}\right)=k\left(u_{2}+u_{3}\right) / 2$, where $k \in[0,1)$;

7. $\phi_{7}\left(u_{1}, u_{2}, u_{3}, u_{4}\right)=k \max \left\{u_{1},\left(u_{2}+u_{3}\right) / 2, u_{4}\right\}$, where $k \in[0,1)$;

8. $\phi_{8}\left(u_{1}, u_{2}, u_{3}, u_{4}\right)=a u_{1}+b\left(u_{2}+u_{3}\right)+2 c u_{4}$, where $a, b, c$ are nonnegative real numbers such that $a+2 b+2 c \in[0,1)$;

9. $\phi_{9}\left(u_{1}, u_{2}, u_{3}, u_{4}\right)=a u_{2}+b u_{3}+c u_{1}$, where $a, b, c$ are nonnegative real numbers such that $a+b+c \in[0,1)$;

10. $\phi_{10}\left(u_{1}, u_{2}, u_{3}, u_{4}\right)=\psi\left(\max \left\{u_{1}, u_{2}, u_{3}, u_{4}\right\}\right)$, where $\psi \in \Psi$.

Lemma 1. (See [2].) Let $(X, d)$ be a metric space and $B \in C L(X)$. Then for each $x \in X$ with $d(x, B)>0$ and $q>1$, there exists an element $b \in B$ such that

$$
d(x, b)<q d(x, B)
$$

Definition 3. Let $(X, d)$ be a metric space. A map $T: X \rightarrow C L(X)$ is said to be an $\alpha$ - $\phi$-contractive mapping if there exist $\alpha: X \times X \rightarrow[0, \infty)$ and $\phi \in \Phi$ such that

$$
H(T x, T y) \leqslant \phi\left(d(x, y), D(x, T x), D(y, T y), \frac{1}{2}(D(y, T x)+D(x, T y))\right)
$$

for all $x, y \in X$ with $\alpha(x, y) \geqslant 1$.

Theorem 2. Let $(X, d)$ be a complete metric space, and let $T: X \rightarrow C L(X)$ be an $\alpha-\phi$ contractive map satisfying the following conditions:

(i) $T$ is $\alpha$-admissible;

(ii) There exist $x_{0} \in X$ and $x_{1} \in T x_{0}$ such that $\alpha\left(x_{0}, x_{1}\right) \geqslant 1$.

Also suppose that one of the following conditions holds:

(iii-a) $T$ is continuous;

(iii-b) If $\left\{x_{n}\right\}$ is a sequence in $X$ such that $x_{n} \rightarrow x$ as $n \rightarrow \infty$ and $\alpha\left(x_{n}, x_{n+1}\right) \geqslant 1$ for each $n \in \mathbb{N}$, then $\alpha\left(x_{n}, x\right) \geqslant 1$ for each $n \in \mathbb{N}$.

Then T has a fixed point. 
Proof. By hypothesis, we have $x_{0} \in X$ and $x_{1} \in T x_{0}$ such that $\alpha\left(x_{0}, x_{1}\right) \geqslant 1$. Suppose $x_{0} \neq x_{1}$, otherwise $x_{0}$ is a fixed point. From (1) we have

$$
\begin{aligned}
& H\left(T x_{0}, T x_{1}\right) \\
& \quad \leqslant \phi\left(d\left(x_{0}, x_{1}\right), D\left(x_{0}, T x_{0}\right), D\left(x_{1}, T x_{1}\right), \frac{1}{2}\left(D\left(x_{1}, T x_{0}\right)+D\left(x_{0}, T x_{1}\right)\right)\right) .
\end{aligned}
$$

For $q>1$, there exists $x_{2} \in T x_{1}$, suppose $x_{2} \neq x_{1}$, otherwise $x_{1}$ is a fixed point, such that we have

$$
0<d\left(x_{1}, x_{2}\right)<q H\left(T x_{0}, T x_{1}\right) .
$$

From (2), since $\phi$ is nondecreasing in each coordinate, we get

$$
H\left(T x_{0}, T x_{1}\right) \leqslant \phi\left(d\left(x_{0}, x_{1}\right), d\left(x_{0}, x_{1}\right), d\left(x_{1}, x_{2}\right), \frac{1}{2}\left(0+d\left(x_{0}, x_{2}\right)\right)\right) .
$$

By triangular inequality, we have

$$
d\left(x_{0}, x_{2}\right) \leqslant d\left(x_{0}, x_{1}\right)+d\left(x_{1}, x_{2}\right) .
$$

We claim that $d\left(x_{1}, x_{2}\right)<d\left(x_{0}, x_{1}\right)$. Suppose, on the contrary, that $d\left(x_{1}, x_{2}\right) \geqslant d\left(x_{0}, x_{1}\right)$, then from (5) we have $d\left(x_{0}, x_{2}\right) \leqslant 2 d\left(x_{1}, x_{2}\right)$. Using these relations in (4), we have

$$
H\left(T x_{0}, T x_{1}\right) \leqslant \phi\left(d\left(x_{1}, x_{2}\right), d\left(x_{0}, x_{1}\right), d\left(x_{1}, x_{2}\right), d\left(x_{1}, x_{2}\right)\right) .
$$

By property (iii) of $\phi$ and (3), we deduce that $H\left(T x_{0}, T x_{1}\right)=0$, which is a contradiction to (3). It follows that $d\left(x_{1}, x_{2}\right)<d\left(x_{0}, x_{1}\right)$, and hence, from (4) we get

$$
H\left(T x_{0}, T x_{1}\right) \leqslant \phi\left(d\left(x_{0}, x_{1}\right), d\left(x_{0}, x_{1}\right), d\left(x_{1}, x_{2}\right), d\left(x_{0}, x_{1}\right)\right) .
$$

By property (ii) of $\phi$, we obtain

$$
H\left(T x_{0}, T x_{1}\right) \leqslant \psi\left(d\left(x_{0}, x_{1}\right)\right) .
$$

Now, from (3) and (6) we have

$$
d\left(x_{1}, x_{2}\right)<q \psi\left(d\left(x_{0}, x_{1}\right)\right) .
$$

Since $\psi$ is strictly increasing, we write

$$
\psi\left(d\left(x_{1}, x_{2}\right)\right)<\psi\left(q \psi\left(d\left(x_{0}, x_{1}\right)\right)\right) .
$$

Let $q_{1}=\psi\left(q \psi\left(d\left(x_{0}, x_{1}\right)\right)\right) / \psi\left(d\left(x_{1}, x_{2}\right)\right)>1$. Since $T$ is $\alpha$-admissible and $x_{2} \in T x_{1}$, $\alpha\left(x_{0}, x_{1}\right) \geqslant 1$ implies $\alpha\left(x_{1}, x_{2}\right) \geqslant 1$; thus, from (1) we have

$$
\begin{aligned}
& H\left(T x_{1}, T x_{2}\right) \\
& \quad \leqslant \phi\left(d\left(x_{1}, x_{2}\right), D\left(x_{1}, T x_{1}\right), D\left(x_{2}, T x_{2}\right), \frac{1}{2}\left(D\left(x_{2}, T x_{1}\right)+D\left(x_{1}, T x_{2}\right)\right)\right) .
\end{aligned}
$$


Since $q_{1}>1$, there exists $x_{3} \in T x_{2}$, suppose $x_{3} \neq x_{2}$, otherwise $x_{2}$ is a fixed point, such that

$$
0<d\left(x_{2}, x_{3}\right)<q_{1} H\left(T x_{1}, T x_{2}\right) .
$$

From (7), since $\phi$ is nondecreasing in each coordinate, we have

$$
H\left(T x_{1}, T x_{2}\right) \leqslant \phi\left(d\left(x_{1}, x_{2}\right), d\left(x_{1}, x_{2}\right), d\left(x_{2}, x_{3}\right), \frac{1}{2}\left(0+d\left(x_{1}, x_{3}\right)\right)\right) .
$$

By triangular inequality, we write

$$
d\left(x_{1}, x_{3}\right) \leqslant d\left(x_{1}, x_{2}\right)+d\left(x_{2}, x_{3}\right) .
$$

We claim that $d\left(x_{2}, x_{3}\right)<d\left(x_{1}, x_{2}\right)$. Suppose, on the contrary, that $d\left(x_{2}, x_{3}\right) \geqslant d\left(x_{1}, x_{2}\right)$; then from (10) we have $d\left(x_{1}, x_{3}\right) \leqslant 2 d\left(x_{2}, x_{3}\right)$. Using these relations in (9), we get

$$
H\left(T x_{1}, T x_{2}\right) \leqslant \phi\left(d\left(x_{2}, x_{3}\right), d\left(x_{1}, x_{2}\right), d\left(x_{2}, x_{3}\right), d\left(x_{2}, x_{3}\right)\right) .
$$

By property (iii) of $\phi$ and (8), we deduce that $H\left(T x_{1}, T x_{2}\right)=0$, which is a contradiction to (8). Therefore, $d\left(x_{2}, x_{3}\right)<d\left(x_{1}, x_{2}\right)$. Next, from (9) we have

$$
H\left(T x_{1}, T x_{2}\right) \leqslant \phi\left(d\left(x_{1}, x_{2}\right), d\left(x_{1}, x_{2}\right), d\left(x_{2}, x_{3}\right), d\left(x_{1}, x_{2}\right)\right)
$$

and, by property (ii) of $\phi$, we obtain

$$
H\left(T x_{1}, T x_{2}\right) \leqslant \psi\left(d\left(x_{1}, x_{2}\right)\right)
$$

From (8) and (11) we have

$$
d\left(x_{2}, x_{3}\right)<q_{1} \psi\left(d\left(x_{1}, x_{2}\right)\right)=\psi\left(q \psi\left(d\left(x_{0}, x_{1}\right)\right)\right),
$$

and hence, since $\psi$ is strictly increasing, we get

$$
\psi\left(d\left(x_{2}, x_{3}\right)\right)<\psi^{2}\left(q \psi\left(d\left(x_{0}, x_{1}\right)\right)\right) .
$$

Let $q_{2}=\psi^{2}\left(q \psi\left(d\left(x_{0}, x_{1}\right)\right)\right) / \psi\left(d\left(x_{2}, x_{3}\right)\right)>1$. Continuing in this way, we construct a sequence $\left\{x_{n}\right\}$ in $X$ such that

$$
x_{n+1} \in T x_{n}, \quad x_{n} \neq x_{n+1}, \quad \alpha\left(x_{n}, x_{n+1}\right) \geqslant 1, \quad d\left(x_{n+1}, x_{n+2}\right)<d\left(x_{n}, x_{n+1}\right)
$$

for all $n \in \mathbb{N} \cup\{0\}$. Further, we have

$$
d\left(x_{n}, x_{n+1}\right)<\psi^{n-1}\left(q \psi\left(d\left(x_{0}, x_{1}\right)\right)\right) \text { for each } n \in \mathbb{N} .
$$

For $n, m \in \mathbb{N}$ with $n>m$, we write

$$
\begin{aligned}
d\left(x_{m}, x_{n}\right) \leqslant & d\left(x_{m}, x_{m+1}\right)+d\left(x_{m+1}, x_{m+2}\right)+\cdots+d\left(x_{n-1}, x_{n}\right) \\
< & \psi^{m-1}\left(q \psi\left(d\left(x_{0}, x_{1}\right)\right)\right)+\psi^{m}\left(q \psi\left(d\left(x_{0}, x_{1}\right)\right)\right)+\cdots \\
& +\psi^{n-2}\left(q \psi\left(d\left(x_{0}, x_{1}\right)\right)\right) \\
= & \sum_{i=m-1}^{n-2} \psi^{i}\left(q \psi\left(d\left(x_{0}, x_{1}\right)\right)\right) \leqslant \sum_{i=m-1}^{\infty} \psi^{i}\left(q \psi\left(d\left(x_{0}, x_{1}\right)\right)\right) .
\end{aligned}
$$


Thus, the convergence of the series $\sum_{i=1}^{\infty} \psi^{i}\left(q \psi\left(d\left(x_{0}, x_{1}\right)\right)\right)$ implies $d\left(x_{n}, x_{m}\right) \rightarrow 0$ as $m \rightarrow \infty$. Therefore, $\left\{x_{n}\right\}$ is a Cauchy sequence in $X$, and, since $X$ is complete, there exists $x^{*} \in X$ such that $x_{n} \rightarrow x^{*}$ as $n \rightarrow \infty$.

Now, if (iii-a) holds, that is, $T$ is continuous, then $x^{*} \in T x^{*}$. In fact, we have

$$
D\left(x^{*}, T x^{*}\right) \leqslant d\left(x^{*}, x_{n+1}\right)+D\left(x_{n+1}, T x^{*}\right) \leqslant d\left(x^{*}, x_{n+1}\right)+H\left(T x_{n}, T x^{*}\right)
$$

and, letting $n \rightarrow \infty$, we obtain $D\left(x^{*}, T x^{*}\right)=0$ and hence $x^{*} \in T x^{*}$. On the other hand, suppose that (iii-b) holds. From (1) we have

$$
\begin{aligned}
D & \left(x_{n+1}, T x^{*}\right) \\
& \leqslant H\left(T x_{n}, T x^{*}\right) \\
& \leqslant \phi\left(d\left(x_{n}, x^{*}\right), D\left(x_{n}, T x_{n}\right), D\left(x^{*}, T x^{*}\right), \frac{1}{2}\left(D\left(x^{*}, T x_{n}\right)+D\left(x_{n}, T x^{*}\right)\right)\right) \\
& \leqslant \phi\left(d\left(x_{n}, x^{*}\right), d\left(x_{n}, x_{n+1}\right), D\left(x^{*}, T x^{*}\right), \frac{1}{2}\left(d\left(x^{*}, x_{n+1}\right)+D\left(x_{n}, T x^{*}\right)\right)\right) .
\end{aligned}
$$

Since $\phi$ is continuous, letting $n \rightarrow \infty$ in (12), we get

$$
D\left(x^{*}, T x^{*}\right) \leqslant \phi\left(0,0, D\left(x^{*}, T x^{*}\right), \frac{D\left(x^{*}, T x^{*}\right)}{2}\right) .
$$

By property (iv) of $\phi$, we deduce that $D\left(x^{*}, T x^{*}\right)=0$. By closedness of $T x^{*}$, we have $x^{*} \in T x^{*}$.

\section{Further discussion and example}

\section{1 $\alpha_{*}$-admissible maps}

We give some results for an $\alpha_{*}$-admissible map in the setting of metric spaces.

Corollary 1. Let $(X, d)$ be a complete metric space, and let $T: X \rightarrow C L(X)$ be a map. Assume there exist $\alpha: X \times X \rightarrow[0, \infty)$ and $\phi \in \Phi$ such that

(i) $\alpha(x, y) H(T x, T y) \leqslant \phi(d(x, y), D(x, T x), D(y, T y),(D(y, T x)+D(x, T y)) / 2)$ for all $x, y \in X$

(ii) $T$ is $\alpha_{*}$-admissible;

(iii) There exist $x_{0} \in X$ and $x_{1} \in T x_{0}$ such that $\alpha\left(x_{0}, x_{1}\right) \geqslant 1$.

Also suppose that one of the following conditions holds:

(iv-a) $T$ is continuous;

(iv-b) If $\left\{x_{n}\right\}$ is a sequence in $X$ such that $x_{n} \rightarrow x$ as $n \rightarrow \infty$ and $\alpha\left(x_{n}, x_{n+1}\right) \geqslant 1$ for each $n \in \mathbb{N}$, then $\alpha\left(x_{n}, x\right) \geqslant 1$ for each $n \in \mathbb{N}$.

Then $T$ has a fixed point.

Proof. Each $\alpha_{*}$-admissible map is obviously $\alpha$-admissible, and hence, the conclusion follows from Theorem 2 . 
Moreover, we give the following theorem.

Theorem 3. Let $(X, d)$ be a complete metric space, and let $T: X \rightarrow C L(X)$ be a map. Assume there exist $\alpha: X \times X \rightarrow[0, \infty)$ and $\phi \in \Phi$ such that

(i) $H(T x, T y) \leqslant \phi(d(x, y), D(x, T x), D(y, T y),(D(y, T x)+D(x, T y)) / 2)$ for all $x, y \in X$ with $\alpha_{*}(T x, T y) \geqslant 1$;

(ii) $T$ is $\alpha_{*}$-admissible;

(iii) There exist $x_{0} \in X$ and $x_{1} \in T x_{0}$ such that $\alpha\left(x_{0}, x_{1}\right) \geqslant 1$.

Also suppose that one of the following conditions holds:

(iv-a) $T$ is continuous;

(iv-b) If $\left\{x_{n}\right\}$ is a sequence in $X$ such that $x_{n} \rightarrow x$ as $n \rightarrow \infty$ and $\alpha\left(x_{n}, x_{n+1}\right) \geqslant 1$ for each $n \in \mathbb{N}$, then $\alpha\left(x_{n}, x\right) \geqslant 1$ for each $n \in \mathbb{N}$.

Then T has a fixed point.

Proof. The proof of this theorem runs on the same lines as the proof of Theorem 2, and hence, to avoid repetition, the details are omitted.

Next corollary follows from the above result.

Corollary 2. Let $(X, d)$ be a complete metric space, and let $T: X \rightarrow C L(X)$ be a map. Assume there exist $\alpha: X \times X \rightarrow[0, \infty)$ and $\phi \in \Phi$ such that

(i) $\alpha_{*}(T x, T y) H(T x, T y) \leqslant \phi(d(x, y), D(x, T x), D(y, T y),(D(y, T x)+D(x$, $T y)) / 2$ ) for all $x, y \in X$;

(ii) $T$ is $\alpha_{*}$-admissible;

(iii) There exist $x_{0} \in X$ and $x_{1} \in T x_{0}$ such that $\alpha\left(x_{0}, x_{1}\right) \geqslant 1$.

Also suppose that one of the following conditions holds:

(iv-a) $T$ is continuous;

(iv-b) If $\left\{x_{n}\right\}$ is a sequence in $X$ such that $x_{n} \rightarrow x$ as $n \rightarrow \infty$ and $\alpha\left(x_{n}, x_{n+1}\right) \geqslant 1$ for each $n \in \mathbb{N}$, then $\alpha\left(x_{n}, x\right) \geqslant 1$ for each $n \in \mathbb{N}$.

Then T has a fixed point.

Remark 1. Note that [7, Thm. 2.1] follows from above corollary.

We give an illustrative example of Corollary 1 and hence of Theorem 2.

Example 1. Let $X=[0, \infty)$ be endowed with the usual metric $d(x, y)=|x-y|$ for all $x, y \in X$. Define $T: X \rightarrow C L(X)$ by

$$
T x= \begin{cases}{[0, x / 8]} & \text { if } x \in[0,4), \\ \{0\} & \text { if } x=4, \\ {\left[0, \mathrm{e}^{x}\right]} & \text { if } x>4\end{cases}
$$


and define $\alpha: X \times X \rightarrow[0, \infty)$ by

$$
\alpha(x, y)= \begin{cases}2 & \text { if } x, y \in[0,4] \\ d(x, y) /\left(2\left(\mathrm{e}^{x}+\mathrm{e}^{y}\right)\right) & \text { otherwise }\end{cases}
$$

Further, consider $\phi\left(u_{1}, u_{2}, u_{3}, u_{4}\right)=\psi\left(\max \left\{u_{1}, u_{3}\right\}\right)$ with $\psi(t)=t / 2$ for each $t \geqslant 0$. Clearly, $\phi \in \Phi$. First, we show that $T$ satisfies condition (i) of Corollary 1 . We distinguish the following cases:

Case 1. If $x, y \in[0,4)$, then we have

$$
\alpha(x, y) H(T x, T y)=\frac{1}{4}|x-y| \leqslant \frac{1}{2}|x-y| \leqslant \psi(\max \{d(x, y), D(y, T y)\}) .
$$

Case 2. If $x \in[0,4)$ and $y=4$, then we have

$$
\alpha(x, y) H(T x, T y)=\frac{x}{4}<1 \leqslant \psi(\max \{d(x, y), D(y, T y)\}) .
$$

Case 3. If $x \in[0,4)$ and $y>4$, then we have

$$
\begin{aligned}
& \alpha(x, y) H(T x, T y) \\
& \quad=\frac{d(x, y)}{2\left(\mathrm{e}^{x}+\mathrm{e}^{y}\right)}\left|\frac{x}{8}-\mathrm{e}^{y}\right|<\frac{d(x, y)}{2} \leqslant \psi(\max \{d(x, y), D(y, T y)\}) .
\end{aligned}
$$

Case 4. If $x=4$ and $y>4$, then we have

$$
\begin{aligned}
& \alpha(x, y) H(T x, T y) \\
& \quad=\frac{d(x, y)}{2\left(e^{x}+e^{y}\right)}\left|0-\mathrm{e}^{y}\right|<\frac{d(x, y)}{2} \leqslant \psi(\max \{d(x, y), D(y, T y)\}) .
\end{aligned}
$$

Case 5. If $x, y>4$, then we have

$$
\begin{aligned}
& \alpha(x, y) H(T x, T y) \\
& =\frac{d(x, y)}{2\left(\mathrm{e}^{x}+\mathrm{e}^{y}\right)}\left|\mathrm{e}^{x}-\mathrm{e}^{y}\right|<\frac{d(x, y)}{2} \leqslant \psi(\max \{d(x, y), D(y, T y)\}) .
\end{aligned}
$$

Thus, condition (i) of Corollay 1 holds true. Notice that $\alpha(x, y) \geqslant 1$ only for $x, y \in[0,4]$; this implies $\alpha_{*}(T x, T y) \geqslant 1$ for $x, y \in[0,4]$, and hence, $T$ is $\alpha_{*}$-admissible. For $x_{0}=1$, we have $x_{1}=1 / 8 \in T x_{0}$ such that $\alpha\left(x_{0}, x_{1}\right)=2$. Moreover, for any sequence $\left\{x_{n}\right\} \subseteq X$ such that $x_{n} \rightarrow x$ as $n \rightarrow \infty$ and $\alpha\left(x_{n}, x_{n+1}\right) \geqslant 1$ for each $n \in \mathbb{N}$, we have $\alpha\left(x_{n}, x\right) \geqslant 1$ for each $n \in \mathbb{N}$. As all the conditions of Corollary 1 (Theorem 2) hold, we conclude that $T$ has a fixed point. 


\subsection{Some consequences}

As consequences of Theorem 2, we give some corollaries. In particular, various theorems of the existing literature can be easily derived by our main theorem.

Corollary 3. Let $(X, d)$ be a complete metric space, and let $T: X \rightarrow C L(X)$ be a map. Assume there exists $\phi \in \Phi$ such that

$$
H(T x, T y) \leqslant \phi\left(d(x, y), D(x, T x), D(y, T y), \frac{1}{2}(D(y, T x)+D(x, T y))\right)
$$

for all $x, y \in X$. Then $T$ has a fixed point.

Proof. Let $\alpha(x, y)=1$ for all $x, y \in X$. Therefore, the conclusion follows from Corollary 1 (Theorem 2).

Corollary 4. Let $(X, d)$ be a complete metric space, and let $T: X \rightarrow C L(X)$ be a map. Assume there exist $\alpha: X \times X \rightarrow[0, \infty)$ and $\psi \in \Psi$ such that

(i) $H(T x, T y) \leqslant \psi(\max \{d(x, y), D(x, T x), D(y, T y),(D(y, T x)+D(x, T y)) / 2\})$ for all $x, y \in X$ with $\alpha(x, y) \geqslant 1$;

(ii) $T$ is $\alpha$-admissible;

(iii) There exist $x_{0} \in X$ and $x_{1} \in T x_{0}$ such that $\alpha\left(x_{0}, x_{1}\right) \geqslant 1$.

Also suppose that one of the following conditions holds:

(iv-a) $T$ is continuous;

(iv-b) If $\left\{x_{n}\right\}$ is a sequence in $X$ such that $x_{n} \rightarrow x$ as $n \rightarrow \infty$ and $\alpha\left(x_{n}, x_{n+1}\right) \geqslant 1$ for each $n \in \mathbb{N}$, then $\alpha\left(x_{n}, x\right) \geqslant 1$ for each $n \in \mathbb{N}$.

Then T has a fixed point.

Proof. Let $\phi\left(u_{1}, u_{2}, u_{3}, u_{4}\right)=\phi_{10}\left(u_{1}, u_{2}, u_{3}, u_{4}\right)=\psi\left(\max \left\{u_{1}, u_{2}, u_{3}, u_{4}\right\}\right)$, where $\psi \in \Psi$. Thus, the conclusion follows from Theorem 2 .

Remark 2. Note that [6, Thm. 2.2] is a special case of above corollary.

Corollary 5. (See [21].) Let $(X, d)$ be a complete metric space, and let $T: X \rightarrow C B(X)$ be a map such that

$$
H(T x, T y) \leqslant k d(x, y)
$$

for all $x, y \in X$, where $k \in[0,1)$. Then $T$ has a fixed point.

Proof. Let $\alpha(x, y)=1$ for all $x, y \in X$ and $\phi\left(u_{1}, u_{2}, u_{3}, u_{4}\right)=\phi_{5}\left(u_{1}, u_{2}, u_{3}, u_{4}\right)=$ $k u_{1}$, where $k \in[0,1)$. Then from (1) we have

$$
H(T x, T y) \leqslant k d(x, y)
$$

for each $x, y \in X$. Therefore, by an application of Theorem 2, we deduce that $T$ has a fixed point. 
Corollary 6. (See [23].) Let $(X, d)$ be a complete metric space, and let $T: X \rightarrow C B(X)$ be a map such that

$$
H(T x, T y) \leqslant k(D(x, T x)+D(y, T y))
$$

for all $x, y \in X$, where $k \in[0,1 / 2)$. Then $T$ has a fixed point.

Proof. Let $\alpha(x, y)=1$ for all $x, y \in X$ and $\phi\left(u_{1}, u_{2}, u_{3}, u_{4}\right)=\phi_{6}\left(u_{1}, u_{2}, u_{3}, u_{4}\right)=$ $\left(\beta\left(u_{2}+u_{3}\right) / 2\right.$, where $\beta \in[0,1)$. Then from (1) we have

$$
H(T x, T y) \leqslant \frac{\beta}{2}(D(x, T x)+D(y, T y))=k(D(x, T x)+D(y, T y))
$$

for each $x, y \in X$, where $k \in[0,1 / 2)$. Therefore, by an application of Theorem 2, we deduce that $T$ has a fixed point.

Corollary 7. (See [11].) Let $(X, d)$ be a complete metric space, and let $T: X \rightarrow C B(X)$ be a map such that

$$
H(T x, T y) \leqslant a d(x, y)+b(D(x, T x)+D(y, T y))+c(D(y, T x)+D(x, T y))
$$

for all $x, y \in X$, where $a, b, c$ are nonnegative real numbers such that $a+2 b+2 c \in[0,1)$. Then $T$ has a fixed point.

Proof. Let $\alpha(x, y)=1$ for all $x, y \in X$ and $\phi\left(u_{1}, u_{2}, u_{3}, u_{4}\right)=\phi_{8}\left(u_{1}, u_{2}, u_{3}, u_{4}\right)=$ $a u_{1}+b\left(u_{2}+u_{3}\right)+2 c u_{4}$, where $a, b, c$ are nonnegative real numbers such that $a+2 b+2 c \in$ $[0,1)$. Then from (1) we have

$$
H(T x, T y) \leqslant a d(x, y)+b(D(x, T x)+D(y, T y))+c(D(y, T x)+D(x, T y)) .
$$

Therefore, by an application of Theorem 2, we deduce that $T$ has a fixed point.

Corollary 8. (See [11].) Let $(X, d)$ be a complete metric space, and let $T: X \rightarrow C B(X)$ be a map such that

$$
H(T x, T y) \leqslant k(D(y, T x)+D(x, T y))
$$

for all $x, y \in X$, where $k \in[0,1 / 2)$. Then $T$ has a fixed point.

Proof. Let $\alpha(x, y)=1$ for all $x, y \in X$ and $\phi\left(u_{1}, u_{2}, u_{3}, u_{4}\right)=\phi_{2}\left(u_{1}, u_{2}, u_{3}, u_{4}\right)=$ $\beta u_{4}$, where $\beta \in[0,1)$. Then from (1) we have

$$
H(T x, T y) \leqslant \frac{\beta}{2}(D(y, T x)+D(x, T y))=k(D(y, T x)+D(x, T y))
$$

for all $x, y \in X$, where $k \in[0,1 / 2)$. Therefore, by an application of Theorem 2, we deduce that $T$ has a fixed point. 
Remark 3. The family of functions $\Phi$ can be extended by replacing function $\psi$ with the $M T$-function introduced by Mizoguchi and Takahashi [19] or with the GMT-function introduced by Javahernia et al. [13]. Moreover, by using the contractive condition

$$
D(y, T y) \leqslant \phi\left(d(x, y), D(x, T x), D(y, T y), \frac{1}{2}(D(y, T x)+D(x, T y))\right)
$$

for all $x \in X$ and $y \in T x$ with $\alpha(x, y) \geqslant 1$, instead of condition (1) in Theorem 2 with $\Phi$ defined along with $M T$-function/ $G M T$-function, we are able to generalize either completely or partially many other results of the literature including the results mentioned in $[1,5,13,15,16,18,19]$.

\subsection{Ordered metric spaces}

Let $(X, \preccurlyeq, d)$ be an ordered metric space and $A, B \subseteq X$. We say that $A \prec_{r} B$ if, for each $a \in A$ and $b \in B$, we have $a \preccurlyeq b$. Also, $A \preccurlyeq B$ whenever, for each $a \in A$, there exists $b \in B$ such that $a \preccurlyeq b$. Notice that the function $\alpha: X \times X \rightarrow[0, \infty)$, defined by

$$
\alpha(x, y)= \begin{cases}1 & \text { if } x \preccurlyeq y, \\ 0 & \text { otherwise }\end{cases}
$$

is an easy example of function suitable for Theorem 2. By using opportunely this fact, we present some results in the setting of ordered metric spaces.

Corollary 9. Let $(X, \preccurlyeq, d)$ be a complete ordered metric space, and let $T: X \rightarrow C L(X)$ be a map such that

(i) $H(T x, T y) \leqslant \phi(d(x, y), D(x, T x), D(y, T y),(D(y, T x)+D(x, T y)) / 2)$ for all $x, y \in X$ with $x \preccurlyeq y$ and $\phi \in \Phi$;

(ii) If $x \preccurlyeq y$, then $T x \prec_{r} T y$;

(iii) There exist $x_{0} \in X$ and $x_{1} \in T x_{0}$ such that $x_{0} \preccurlyeq x_{1}$.

Also suppose that one of the following conditions holds:

(iv-a) $T$ is continuous;

(iv-b) If $\left\{x_{n}\right\}$ is a sequence in $X$ such that $x_{n} \rightarrow x$ as $n \rightarrow \infty$ and $x_{n} \preccurlyeq x_{n+1}$ for each $n \in \mathbb{N}$, then $x_{n} \preccurlyeq x$ for each $n \in \mathbb{N}$.

Then $T$ has a fixed point.

Proof. Define $\alpha: X \times X \rightarrow[0, \infty)$ by

$$
\alpha(x, y)= \begin{cases}1 & \text { if } x \preccurlyeq y \\ 0 & \text { otherwise }\end{cases}
$$

Thus, it is easy to see that all conditions of Theorem 2 hold, and hence, $T$ has a fixed point. 
Corollary 10. Let $(X, \preccurlyeq, d)$ be a complete ordered metric space, and let $T: X \rightarrow$ $C L(X)$ be a map such that

(i) $H(T x, T y) \leqslant \phi(d(x, y), D(x, T x), D(y, T y),(D(y, T x)+D(x, T y)) / 2)$ for all $x, y \in X$ with $T x \preccurlyeq T y$ and $\phi \in \Phi$;

(ii) If, for each $x \in X$ and $y \in T x, T x \preccurlyeq T y$, then $T y \preccurlyeq T z$ for all $z \in T y$;

(iii) There exist $x_{0} \in X$ and $x_{1} \in T x_{0}$ such that $T x_{0} \preccurlyeq T x_{1}$.

Also suppose that one of the following conditions holds:

(iv-a) $T$ is continuous;

(iv-b) If $\left\{x_{n}\right\}$ is a sequence in $X$ such that $x_{n} \rightarrow x$ as $n \rightarrow \infty$ and $T x_{n} \preccurlyeq T x_{n+1}$ for each $n \in \mathbb{N}$, then $T x_{n} \preccurlyeq T x$ for each $n \in \mathbb{N}$.

Then $T$ has a fixed point.

Proof. Define the function $\alpha: X \times X \rightarrow[0, \infty)$ by

$$
\alpha(x, y)= \begin{cases}1 & \text { if } T x \preccurlyeq T y, \\ 0 & \text { otherwise. }\end{cases}
$$

Clearly, the multivalued map $T$ is $\alpha$-admissible. In fact, for each $x \in X$ and $y \in T x$ with $\alpha(x, y) \geqslant 1$, we have $T x \preccurlyeq T y$, and, by condition (ii), we obtain that $T y \preccurlyeq T z$ for all $z \in T y$. This implies that $\alpha(y, z) \geqslant 1$ for all $z \in T y$. Thus, all the hypotheses of Theorem 2 are satisfied, and $T$ has a fixed point.

\section{Application to integral equation}

Based on our fixed point results, this section is dedicated to establishing the existence of solutions for integral equations. First, we give the background and notation. Let $X=$ $C([0, I], \mathbb{R})$ be the set of continuous real functions on $[0, I]$ with $I>0$, and $d: X \times X \rightarrow$ $[0, \infty)$ be given by

$$
d(x, y)=\|x-y\|_{\infty} \quad \text { for all } x, y \in X .
$$

Then $(X, d)$ is a complete metric space. Also, consider the integral equation

$$
x(t)=p(t)+\int_{0}^{I} S(t, u) f(u, x(u)) \mathrm{d} u,
$$

where $f:[0, I] \times \mathbb{R} \rightarrow \mathbb{R}, p:[0, I] \rightarrow \mathbb{R}$ are two continuous functions and $S:[0, I] \times$ $[0, I] \rightarrow[0, \infty)$ is a function such that $S(t, \cdot) \in L^{1}([0, I])$ for all $t \in[0, I]$.

Then we prove the following theorem.

Theorem 4. Let $X=C([0, I], \mathbb{R})$, and let $T: X \rightarrow X$ be the operator defined by

$$
T(x)(t)=p(t)+\int_{0}^{I} S(t, u) f(u, x(u)) \mathrm{d} u .
$$


Assume there exist $\alpha, \beta: X \times X \rightarrow[0, \infty)$ such that

(i) If $\alpha(x, y) \geqslant 1$ for $x, y \in X$, then, for every $u \in[0, I]$ and some $k \in(0,1)$, we have

$$
0 \leqslant|f(u, x(u))-f(u, y(u))| \leqslant \beta(x, y)|x(u)-y(u)|
$$

and

$$
\left\|\int_{0}^{I} S(t, u) \beta(x, y) \mathrm{d} u\right\|_{\infty} \leqslant k
$$

(ii) $x, y \in X, \alpha(x, y) \geqslant 1$ implies $\alpha(T x, T y) \geqslant 1$;

(iii) There exists $x_{0} \in X$ such that $\alpha\left(x_{0}, T\left(x_{0}\right)\right) \geqslant 1$;

(iv) If $\left\{x_{n}\right\}$ is a sequence in $X$ such that $\alpha\left(x_{n}, x_{n+1}\right) \geqslant 1$ for each $n \in \mathbb{N}$ and $x_{n} \rightarrow x$ as $n \rightarrow \infty$, then $\alpha\left(x_{n}, x\right) \geqslant 1$ for each $n \in \mathbb{N}$.

Then the integral equation (13) has a solution in $X$.

Proof. Notice that any fixed point of the integral operator (14) is a solution of (13). Then, by condition (i), we obtain

$$
\begin{aligned}
|T(x)(t)-T(y)(t)| & =\left|\int_{0}^{I} S(t, u)[f(u, x(u))-f(u, y(u))] \mathrm{d} u\right| \\
& \leqslant \int_{0}^{I} S(t, u)|f(u, x(u))-f(u, y(u))| \mathrm{d} u \\
& \leqslant \int_{0}^{I} S(t, u) \beta(x, y)|x(u)-y(u)| \mathrm{d} u \\
& \leqslant\|x-y\|_{\infty} \int_{0}^{I} S(t, u) \beta(x, y) \mathrm{d} u .
\end{aligned}
$$

Consequently, we have

$$
\|T(x)-T(y)\|_{\infty} \leqslant\|x-y\|_{\infty}\left\|\int_{0}^{I} S(t, u) \beta(x, y) \mathrm{d} u\right\|_{\infty},
$$

and hence, for all $x, y \in X$ with $\alpha(x, y) \geqslant 1$, we obtain

$$
d(T(x), T(y)) \leqslant k d(x, y) \text {. }
$$

Thus, condition (i) of Corollary 3 is trivially satisfied with $\psi(t)=k t$ for all $t \geqslant 0$. The other conditions of Corollary 3 are immediately satisfied, and hence, the operator $T$ has a fixed point, that is, there exists a solution of the integral equation (13) in $X$. 
Remark 4. Clearly, the function $\alpha: X \times X \rightarrow[0, \infty)$, mentioned at the beginning of Section 3.3 and defined by

$$
\alpha(x, y)= \begin{cases}1 & \text { if } x \preccurlyeq y \\ 0 & \text { otherwise }\end{cases}
$$

is suitable for Theorem 4 .

Acknowledgment. Authors are thankful to the Reviewers for their useful comments to improve the quality of the paper.

\section{References}

1. J. Ahmad, N. Hussain, A.R. Khan, A. Azam, Fixed point results for generalized multi-valued contractions, J. Nonlinear Sci. Appl., 8:909-918, 2015.

2. M.U. Ali, T. Kamran, On $\left(\alpha^{*}, \psi\right)$-contractive multi-valued mappings, Fixed Point Theory Appl., 2013:137, 2013.

3. M.U. Ali, T. Kamran, E. Karapınar, $(\alpha, \psi, \xi)$-contractive multi-valued mappings, Fixed Point Theory Appl., 2014:7, 2014.

4. M.U. Ali, T. Kamran, E. Karapınar, A new approach to $(\alpha, \psi)$-contractive nonself multivalued mappings, J. Inequal. Appl., 2014:71, 2014.

5. M.U. Ali, T. Kamran, W. Sintunavarat, P. Katchang, Mizoguchi-Takahashi's fixed point theorem with $\alpha, \eta$ functions, Abstr. Appl. Anal., 2013:418798, 2013.

6. P. Amiri, S. Rezapour, N. Shahzad, Fixed points of generalized $\alpha-\psi$-contractions, Rev. R. Acad. Cienc. Exactas Fís. Nat., Ser. A Math., RACSAM, 108:519-526, 2014.

7. J.H. Asl, S. Rezapour, N. Shahzad, On fixed points of $\alpha-\psi$-contractive multifunctions, Fixed Point Theory Appl., 2012:212, 2012.

8. V. Berinde, F. Vetro, Common fixed points of mappings satisfying implicit contractive conditions, Fixed Point Theory Appl., 2012:105, 2012.

9. T.H. Chang, Common fixed point theorems for multi-valued mappings, Math. Japon., 41:311320, 1995.

10. P.Z. Daffer, H. Kaneko, Fixed points of generalized contractive multi-valued mappings, J. Math. Anal. Appl., 192:655-666, 1995.

11. M.E. Gordji, H. Banghani, H. Khodaei, M. Ramezani, A generalization of Nadler's fixed point theorem, J. Nonlinear Sci. Appl., 3:148-151, 2010.

12. N. Hussain, P. Salimi, A. Latif, Fixed point results for single and set-valued $\alpha-\eta$ - $\psi$-contractive mappings, Fixed Point Theory Appl., 2013:212, 2013.

13. M. Javahernia, A. Razani, F. Khojasteh, Common fixed point of the generalized MizoguchiTakahashi's type contractions, Fixed Point Theory Appl., 2014:195, 2014.

14. M. Jleli, B. Samet, C. Vetro, F. Vetro, Fixed points for multivalued mappings in $b$-metric spaces, Abstr. Appl. Anal., 2015:718074, 2015. 
15. T. Kamran, Mizoguchi-Takahashi's type fixed point theorem, Comput. Math. Appl., 57:507511, 2009.

16. Q. Kiran, M.U. Ali, T. Kamran, Generalization of Mizoguchi-Takahashi type contraction and related fixed point theorems, J. Inequal. Appl., 2014:458, 2014.

17. J.T. Markin, A fixed point theorem for set valued mappings, Bull. Am. Math. Soc., 74:639-640, 1968.

18. G. Minak, I. Altun, Some new generalizations of Mizoguchi-Takahashi type fixed point theorem, J. Inequal. Appl., 2013(493), 2013.

19. N. Mizoguchi, W. Takahashi, Fixed point theorems for multivalued mappings on complete metric space, J. Math. Anal. Appl., 141:177-188, 1989.

20. B. Mohammadi, S. Rezapour, N. Shahzad, Some results on fixed points of $\alpha-\psi$-Ćirić generalized multifunctions, Fixed Point Theory Appl., 2013(24), 2013.

21. N.B. Nadler, Multi-valued contraction mappings, Pac. J. Math., 30:475-488, 1969.

22. G. Minak, O. Acar, I. Altun, Multivalued pseudo-Picard operators and fixed point results, J. Funct. Spaces Appl., 2013:827458, 2013.

23. S. Reich, Kannan's fixed point theorem, Boll. Unione Mat. Ital., IV. Ser., 4:1-11, 1971.

24. B. Samet, C. Vetro, P. Vetro, Fixed point theorems for $\alpha$ - $\psi$-contractive type mappings, Nonlinear Anal., Theory Methods Appl., 75:2154-2165, 2012. 